\title{
Plane Symmetric Bulk Viscous Domain Wall in Lyra Geometry
}

\author{
Anirudh Pradhan ${ }^{1}$, Kanchan Kumar Rai ${ }^{2}$, and Anil Kumar Yadav ${ }^{3}$ \\ ${ }^{1}$ Department of Mathematics, Hindu Post-graduate College, Zamania-232 331, Ghazipur, India \\ 2 Department of Mathematics, Hindu Post-graduate College, Zamania-232 331, Ghazipur, India and \\ ${ }^{3}$ Department of Physics, K. N. Govt. Post-graduate College, \\ Gyanpur, Sant Ravidas Nagar, Bhadohi-221 304, India
}

Received on 14 November, 2006

\begin{abstract}
In this paper general solutions are found for domain walls in Lyra geometry in the plane symmetric spacetime metric given by Taub. Expressions for the energy density and pressure of domain walls are derived in both cases of uniform and time varying displacement field $\beta$. Some physical consequences of the models are also given. Finally, the geodesic equations and acceleration of the test particle are discussed.
\end{abstract}

Keywords: Cosmology; Plane symmetric domain walls; Bulk viscous model; Lyra geometry

\section{INTRODUCTION}

Domain walls form when a discrete symmetry is spontaneously broken [1-5]. In simplest models, symmetry breaking is accomplished by a real scalar field $\phi$ whose vacuum manifold is disconnected. For example, suppose that the scalar potential for $\phi$ is $U(\phi)=\lambda\left(\phi^{2}-\mu^{2}\right)^{2}$. The vacuum manifold for $\phi$ consists of the two points $[\phi=\mu$ and $\phi=-\mu]$. After symmetry breaking, different regions of the universe can settle into different parts of the vacuum with domain walls forming the boundaries between these regions. The stress energy for a static, plane-symmetric domain wall consists of a positive surface energy density and a surface tension equal in magnitude to the surface energy [3]. We note, however that this analysis neglects the effects of gravity [6]. Locally, the stress energy for a wall of arbitrary shape is similar to that of a plane-symmetric wall having both surface energy density and surface tension. Closed-surface domain walls collapse due to their surface tension. However, the details of the collapse for a wall with arbitrary shape and finite thickness are largely unknown.

The spacetime of cosmological domain walls has now been a subject of interest for more than a decade since the work of Vilenkin and Ipser and Sikivie [7, 8] who use Israel's thin wall formalism [9] to compute the gravitational field of an infinitesimally thin planar domain wall. After the original work by Vilenkin, Ipser and Sikivie [7, 8] for thin walls, attempts focused on trying to find a perturbative expansion in the wall thickness $[6,10]$. With the proposition by Hill, Schramn and Fry [11] of a late phase transition with thick domain walls, there was some effort in finding exact thick solution [12, 13]. Recently, Bonjour et al [14] considered gravitating thick domain wall solutions with planar and reflection symmetry in the Goldstone model. Bonjour et al [15] also investigated the spacetime of a thick gravitational domain wall for a general potential $V(\phi)$. Jensen and Soleng [16] have studied anisotropic domain walls where the solution has naked singularities and the generic solution is unstable to Hawking decay.

The investigation of relativistic cosmological models usually has the energy momentum tensor of matter generated by a perfect fluid. To consider more realistic models one must take into account the viscosity mechanisms, which have already attracted the attention of many researchers. Most studies in cosmology involve a perfect fluid. Large entropy per baryon and the remarkable degree of isotropy of the cosmic microwave background radiation, suggest that we should analyze dissipative effects in cosmology. Further, there are several processes which are expected to give rise to viscous effect. These are the decoupling of neutrinos during the radiation era and the recombination era [17], decay of massive super string modes into massless modes [18], gravitational string production $[19,20]$ and particle creation effect in grand unification era [21]. It is known that the introduction of bulk viscosity can avoid the big bang singularity. Thus, we should consider the presence of a material distribution other than a perfect fluid to have realistic cosmological models (see Grøn [22] for a review on cosmological models with bulk viscosity). A uniform cosmological model filled with fluid which possesses pressure and second (bulk) viscosity was developed by Murphy [50]. The solutions that he found exhibit an interesting feature that the big bang type singularity appears in the infinite past.

To study thick domain walls, one can study the field equations as well as equations of the domain walls treated as the self interacting scalar field. A thick domain wall can be viewed as a soliton-like solution of the scalar field equation coupled with gravity. In order to determine the gravitational field one has to solve Einstein's equation with an energy momentum tensor $T_{\mu \nu}$ describing a scalar field $\phi$ with self-interactions contained in a potential $V(\phi)[6-8,12]$.

$$
T_{\mu v}=\delta_{\mu} \phi \delta_{v} \phi-g_{\mu v}\left(\frac{1}{2} \delta_{\sigma} \phi \delta^{\sigma} \phi-V(\phi)\right)
$$

The influence of the viscous fluid in the evolution of the universe is performed by means of its energy momentum tensor, which acts as the source of the corresponding gravitational field. Thick domain walls are characterized by the energy momentum tensor of a viscous field which has the form

$$
T_{i k}=\rho\left(g_{i k}+w_{i} w_{k}\right)+\bar{p} w_{i} w_{k}, \quad w_{i} w^{i}=-1,
$$


where

$$
\bar{p}=p-\xi w_{; i}^{i}
$$

Here $\rho, p, \bar{p}$, and $\xi$ are the energy density, the pressure in the direction normal to the plane of the wall, effective pressure, bulk viscous coefficient respectively and $w_{i}$ is a unit space like vector in the same direction.

In 1951 Lyra [24] proposed a modification of Riemannian geometry by introducing a gauge function into the structureless manifold, as a result of which the cosmological constant arises naturally from geometry. This bears a remarkable resemblance to Weyl's geometry [25]. But in Lyra's geometry, unlike Weyl's, the connection is metric preserving as in Riemannian; in other words, length transfers are integrable. Lyra also introduced the notion of a gauge and in the "normal" gauge the curvature scalar is identical to that of Weyl. In subsequent investigations Sen [26], Sen and Dunn [27] proposed a new scalar-tensor theory of gravitation and constructed an analog of the Einstein field equations based on Lyra's geometry. It is, thus, possible [26] to construct a geometrized theory of gravitation and electromagnetism along the lines of Weyl's "unified" field theory without inconvenience of non-integrability length transfer. Halford [28] has pointed out that the constant vector displacement field $\phi_{i}$ in Lyra's geometry plays the role of cosmological constant $\Lambda$ in the normal general relativistic treatment. It is shown by Halford [29] that the scalar-tensor treatment based on Lyra's geometry predicts the same effects, within observational limits as the Einstein's theory. Several investigators viz. Sen and Vanstone [30], Bhamra [31], Karade and Borikar [32], Kalyanshetti and Wagmode [33], Reddy and Innaiah [34], Beesham [35], Reddy and Venkateswarlu [36], Soleng [37], Singh and Singh [38], Singh and Desikan [39], Pradhan and Vishwakarma [40], Pradhan et al $[41,42]$ have studied cosmological models based on Lyra's manifold with a constant displacement field vector. However, this restriction of the displacement field to be constant is merely one of convenience and there is no a priori reason for it. Soleng [37] has pointed out that the cosmologies based on Lyra's manifold with constant gauge vector $\phi$ will either include a creation field and be equal to Hoyle's creation field cosmology [43]-[45] or contain a special vacuum field which together with the gauge vector term may be considered as a cosmological term. In the latter case the solutions are equal to the general relativistic cosmologies with a cosmological term.

The universe is spherically symmetric and the matter distribution in it is on the whole isotropic and homogeneous. But during the early stages of evolution, it is unlikely that it could have had such a smoothed out picture. Hence we consider plane symmetry which provides an opportunity for the study of inhomogeneity. Motivated by the situations discussed above, in this paper we shall focus upon the problem of establishing a formalism for studying the general solution for domain wall in Lyra geometry in the plane symmetric spacetime metric in presence of bulk viscous in an expanding universe. Expressions for the energy density and pressure of domain walls are obtained in both cases of uniform and time varying displacement field $\beta$. We have also shown that the result obtained by Rahaman et al [23] is a special case of our solutions. This paper is organized as follows. The metric and the field equations are presented in Section II. In Section III we deal with the solution of field equations. The Subsection A contain the solution of uniform displacement field ( $\beta=\beta_{0}$, constant). This section also contain the two different models and also the physical consequences of these models. The Subsection B deal with the solution with time varying displacement field $\left(\beta=\beta_{0} t^{\alpha}\right)$. This subsection also contain two different models and these physical consequences are discussed. The geodesic equations and accelerations of the test particle are discussed in Section IV. Finally in Section $\mathrm{V}$ concluding remarks will be given.

\section{FIELD EQUATIONS}

In this section we shall consider the field equations, in normal gauge for Lyra's manifold, obtained by Sen [26] as

$$
R_{i j}-\frac{1}{2} g_{i j} R+\frac{3}{2} \phi_{i} \phi_{j}-\frac{3}{4} g_{i j} \phi_{k} \phi^{k}=-8 \pi G T_{i j},
$$

for study domain walls. The energy momentum tensor $T_{i j}$ in comoving coordinates for thick domain walls take the form

$$
T_{0}^{0}=T_{2}^{2}=T_{3}^{3}=\rho, T_{1}^{1}=-\bar{p}, T_{1}^{0}=0
$$

and displacement vector $\phi_{i}$ is defined by $\phi_{i}=(0,0,0, \beta)$, where $\beta$ may be considered constant as well as function of time coordinate like cosmological constant in Einstein's theory of gravitation.

We consider the most general plane symmetric spacetime metric suggested by Taub [46]

$$
d s^{2}=e^{A}\left(d t^{2}-d z^{2}\right)-e^{B}\left(d x^{2}+d y^{2}\right)
$$

where $A$ and $B$ are functions of $t$ and $z$.

Using equation (5) the field equations (4) for the metric (6) reduce to

$$
\frac{e^{-A}}{4}\left(-4 B^{\prime \prime}-3 B^{\prime 2}+2 A^{\prime} B^{\prime}\right)+\frac{e^{-A}}{4}\left(\dot{B}^{2}+2 \dot{B} \dot{A}\right)-\frac{3}{4} e^{-A} \beta^{2}=8 \pi \rho,
$$

$$
\frac{e^{-A}}{4}\left(-B^{\prime 2}-2 B^{\prime} A^{\prime}\right)+\frac{e^{-A}}{4}\left(-4 \ddot{B}+3 \dot{B}^{2}-2 \dot{A} \dot{B}\right)+\frac{3}{4} e^{-A} \beta^{2}=-8 \pi \bar{p}
$$

$$
\frac{e^{-A}}{4}\left[-2\left(A^{\prime \prime}+B^{\prime \prime}\right)-B^{\prime 2}\right]+\frac{e^{-A}}{4}\left[2(\ddot{A}+\ddot{B})+\dot{B}^{2}\right]+\frac{3}{4} e^{-A} \beta^{2}=8 \pi \rho,
$$

$$
-\dot{B}^{\prime}+\dot{B}\left(A^{\prime}-B^{\prime}\right)+\dot{A} B^{\prime}=0 .
$$

In order to solve the above set of field equations we assume the separable form of the metric coefficients as follows

$$
A=A_{1}(z)+A_{2}(t), \quad B=B_{1}(z)+B_{2}(t)
$$


From Eqs. (10) and (11), we obtain

$$
\frac{A_{1}^{\prime}}{B_{1}^{\prime}}=\frac{\left(\dot{B}_{2}-\dot{A}_{2}\right)}{B_{2}}=m,
$$

where $m$ is considered as separation constant.

Eq. (12) yields the solution

$$
\begin{gathered}
A_{1}=m B_{1} \\
A_{2}=(1-m) B_{2}
\end{gathered}
$$

Again, subtracting Eq. (9) from Eq. (7) and using Eq. (11), we obtain

$$
A_{1}^{\prime \prime}-B_{1}^{\prime \prime}-B_{1}^{\prime 2}+A_{1}^{\prime} B_{1}^{\prime}=\ddot{A}_{2}+\ddot{B}_{2}-\dot{A}_{2} \dot{B}_{2}+3 \beta^{2}=k,
$$

where $k$ is another separation constant. With the help of Eqs (13) and (14), Eq. (15) may be written as

$$
\begin{gathered}
(m-1)\left[B_{1}^{\prime \prime}+B_{1}^{\prime 2}\right]=k \\
(2-m) \ddot{B}_{2}+(m-1) \dot{B}_{2}^{2}=k-3 \beta^{2}
\end{gathered}
$$

\section{SOLUTIONS OF THE FIELD EQUATIONS}

In this section we shall obtain exact solutions for thick domain walls in different cases.

Using the substitution $u=e^{B_{1}}$ and $a=\frac{k}{1-m}$, Eq. (16) takes the form

$$
u^{\prime \prime}+a u=0
$$

which has the solution

$$
e^{B_{1}}=u=c_{1} \sinh (z \sqrt{|a|})+c_{2} \cosh (z \sqrt{|a|}) \quad \text { when } a<0,
$$

where $c_{1}$ and $c_{2}$ are integrating constants. Eq. (19) represent the general solution of the differential Eq. (18) when $a<$ 0 . It may be noted that Rahaman et al [23] have obtained a particular solution for the case $a<0$ in presence of perfect fluid. Their solution can be obtained from Eq. (19) by taking $c_{1}=0$ and $c_{2}=2$.

Eq. (17) may be written as

$$
\ddot{B}_{2}-\frac{(1-m)}{(2-m)} \dot{B}_{2}^{2}+\frac{3}{(2-m)} \beta^{2}=\frac{k}{2-m} .
$$

Now we shall consider uniform and time varying displacement field $\beta$ separately.

\section{A. Case I: Uniform displacement field ( $\beta=\beta_{0}$, constant)}

By use of the transformation $v=e^{-\frac{(1-m)}{(2-m)} B_{2}}$, Eq. (19) reduces to

$$
\ddot{v}+b v=0,
$$

where

$$
b=\frac{(1-m)\left(k-3 \beta_{0}^{2}\right)}{(2-m)^{2}} .
$$

Again, it can be easily seen that Eq. (21) possesses the solution

$e^{-\frac{(1-m)}{(2-m)} B_{2}}=v=\bar{c}_{1} \sinh (t \sqrt{|b|})+\bar{c}_{2} \cosh (t \sqrt{|b|})$ when $b<0$,

where $\bar{c}_{1}$ and $\bar{c}_{2}$ are integrating constants. Hence the metric coefficients have the explicit forms as

$$
e^{A}=\left\{c_{1} \sinh (z \sqrt{|a|})+c_{2} \cosh (z \sqrt{|a|})\right\}^{m} \times
$$

$\left\{\bar{c}_{1} \sinh (t \sqrt{|b|})+\bar{c}_{2} \cosh (t \sqrt{|b|})\right\}^{(m-2)}$ when $a<0, \quad b<0$,

$$
e^{B}=\left\{c_{1} \sinh (z \sqrt{|a|})+c_{2} \cosh (z \sqrt{|a|})\right\} \times
$$

$\left\{\bar{c}_{1} \sinh (t \sqrt{|b|})+\bar{c}_{2} \cosh (t \sqrt{|b|})\right\}^{-\frac{(2-m)}{(1-m)}} \quad$ when $a<0, \quad b<0$.

With the help of Eqs. (23) and (24), the energy density and pressure can be obtained from Eqs. (7) and (8)

$$
32 \pi \rho=e^{-A}\left[(m+1)|a|\left(\frac{Z_{2}}{Z_{1}}\right)^{2}-4|a|+\right.
$$

$$
\left.\frac{(3-m)(2-m)^{2}}{(1-m)^{2}}|b|\left(\frac{T_{2}}{T_{1}}\right)^{2}-3 \beta_{0}^{2}\right] \quad \text { when } a<0, \quad b<0
$$

$$
32 \pi(p-\xi \theta)=e^{-A}\left[(m+1)|a|\left(\frac{Z_{2}}{Z_{1}}\right)^{2}-\frac{4(2-m)}{(1-m)}|b|+\right.
$$

$\left.\frac{(2-m)\left(2 m^{2}-7 m+2\right)}{(1-m)^{2}}|b|\left(\frac{T_{2}}{T_{1}}\right)^{2}-3 \beta_{0}^{2}\right]$ when $a<0, b<0$,

where

$e^{-A}=\cosh ^{-m}(z \sqrt{|a|}) \cosh ^{2-m}(t \sqrt{|b|}) Z_{1}^{-m} T_{1}^{2-m}$,

$Z_{1}=c_{2}+c_{1} \tanh (z \sqrt{|a|})$,

$Z_{2}=c_{1}+c_{2} \tanh (z \sqrt{|a|})$,

$T_{1}=\bar{c}_{2}+\bar{c}_{1} \tanh (t \sqrt{|b|})$,

$T_{2}=\bar{c}_{1}+\bar{c}_{2} \tanh (t \sqrt{|b|})$.

Here $\xi$, in general, is a function of time. The expression for kinematical parameter expansion $\theta$ is given by

$$
\theta=\frac{(m-2)(3-m) \sqrt{|b|}}{2(1-m)} e^{-\frac{A}{2}}\left(\frac{T_{2}}{T_{1}}\right) .
$$

Thus, given $\xi(t)$ we can solve Eq. (26). In most of the investigations involving bulk viscosity is assumed to be a simple power function of the energy density [47]- [49]:

$$
\xi(t)=\xi_{0} \rho^{n},
$$


where $\xi_{0}$ and $n$ are constants. For small density, $n$ may even be equal to unity as used in Murphy's work for simplicity [50]. If $n=1$, Eq. (28) may correspond to a radiative fluid [51]. However, more realistic models [52] are based on $n$ lying in the regime $0 \leq n \leq \frac{1}{2}$.

For simplicity and realistic models of physical importance, we consider the following two cases $(n=0,1)$.

\section{Model I: solution for $\xi=\xi_{0}$}

When $n=0$, Eq. (28) reduces to $\xi=\xi_{0}=$ constant. Hence in this case Eq. (26), with the use of (27), leads to

$$
32 \pi p=32 \pi T_{3}+e^{-A}\left[(m+1)|a|\left(\frac{Z_{2}}{Z_{1}}\right)^{2}-\frac{4(2-m)}{(1-m)}|b|+\right.
$$

$$
\left.\frac{(2-m)\left(2 m^{2}-7 m+2\right)}{(1-m)^{2}}|b|\left(\frac{T_{2}}{T_{1}}\right)^{2}-3 \beta_{0}^{2}\right]
$$

where

$$
T_{3}=\frac{(m-2)(3-m)}{2(1-m)} \sqrt{|b|} e^{-\frac{A}{2}}\left(\frac{T_{2}}{T_{1}}\right) \xi_{0} .
$$

\section{Model II: solution for $\xi=\xi_{0} \rho$}

When $n=1$, Eq. (28) reduces to $\xi=\xi_{0} \rho$ and hence Eq. (26), with the use of (27), leads to

$$
\begin{aligned}
32 \pi p= & e^{-A}\left[\left\{(m+1)|a|\left(\frac{Z_{2}}{Z_{1}}\right)^{2}-3 \beta_{0}^{2}\right\}\left(T_{3}+1\right)-4\left(|a| T_{3}+\frac{(2-m)}{(1-m)}|b|\right)\right. \\
& \left.+\frac{(2-m)}{(1-m)^{2}}|b|\left(\frac{T_{2}}{T_{1}}\right)^{2}\left\{(3-m)(2-m) T_{3}+2 m^{2}-7 m+2\right\}\right] .
\end{aligned}
$$

\section{Physical Consequences of the models}

From the above results in both models, it is evident that at any instant the domain wall density $\rho$ and pressure $p$ in the perpendicular direction decrease on both sides of the wall away from the symmetry plane and both vanish as $Z \longrightarrow \pm \infty$. The space times in both cases are reflection symmetry with respect to the wall. All these properties are very much expected for a domain wall. It can be also seen that the viscosity, as well as the displacement field $\beta$ exhibit essential influence on the character of the solutions.

The weak and strong energy conditions, we have, in Model

$$
\begin{gathered}
32 \pi(\rho-p)=e^{-A}\left[\frac{(2-m)}{(1-m)^{2}}|b|\left(\frac{T_{2}}{T_{1}}\right)^{2}\left(-m^{2}+2 m+4\right)+\right. \\
\left.4\left\{\frac{(2-m)}{(1-m)}|b|-|a|\right\}\right]-32 \pi T_{3}, \\
32 \pi(\rho+p)=e^{-A\left[\frac{(2-m)}{(1-m)^{2}}|b|\left(\frac{T_{2}}{T_{1}}\right)^{2}\left(3 m^{2}-12 m+8\right)+2(m+1)|a|\left(\frac{Z_{2}}{Z_{1}}\right)^{2}+\right.} \\
\left.\quad-4\left\{\frac{(2-m)}{(1-m)}|b|+|a|\right\}-6 \beta_{0}^{2}\right]+32 \pi T_{3}, \\
32 \pi(\rho-3 p)=e^{-A\left[\frac{(2-m)}{(1-m)^{2}}|b|\left(\frac{T_{2}}{T_{1}}\right)^{2}\left(-5 m^{2}+16 m\right)+4\left\{3 \frac{(2-m)}{(1-m)}|b|-|a|\right\}\right.}
\end{gathered}
$$




$$
\begin{gathered}
\left.-2(m+1)|a|\left(\frac{Z_{2}}{Z_{1}}\right)^{2}+6 \beta_{0}^{2}\right]-96 \pi T_{3}, \\
32 \pi(\rho+3 p)=e^{-A}\left[\frac{(2-m)}{(1-m)^{2}}|b|\left(\frac{T_{2}}{T_{1}}\right)^{2}\left(7 m^{2}-26 m+12\right)+4(m+1)|a|\left(\frac{Z_{2}}{Z_{1}}\right)^{2}\right. \\
\left.-4\left\{|a|+\frac{3(2-m)}{(1-m)}|b|\right\}-12 \beta_{0}^{2}\right]+96 \pi T_{3} .
\end{gathered}
$$

In Model II, we have

$$
\begin{aligned}
& 32 \pi(\rho-p)=e^{-A}\left[\frac{(2-m)}{(1-m)^{2}}|b|\left(\frac{T_{2}}{T_{1}}\right)^{2}\left\{\left(-m^{2}+5 m-6\right)\left(T_{3}-1\right)+2 m^{2}-7 m+2\right\}\right. \\
& \left.-4\left\{|a|\left(T_{3}+1\right)+\frac{(2-m)}{(1-m)}|b|\right\}-(m+1)|a|\left(\frac{Z_{2}}{Z_{1}}\right)^{2} T_{3}+3 \beta_{0}^{2} T_{3}\right], \\
& 32 \pi(\rho+p)=e^{-A}\left[\frac{(2-m)}{(1-m)^{2}}|b|\left(\frac{T_{2}}{T_{1}}\right)^{2}\left\{\left(m^{2}-5 m+6\right)\left(T_{3}+1\right)+2 m^{2}-7 m+2\right\}\right. \\
& \left.+(m+1)|a|\left(\frac{Z_{2}}{Z_{1}}\right)^{2}\left(T_{3}+2\right)-4\left\{|a|\left(T_{3}+1\right)+\frac{(2-m)}{(1-m)}|b|\right\}-3 \beta_{0}^{2}\left(T_{3}+2\right)\right], \\
& 32 \pi(\rho-3 p)=e^{-A}\left[-\frac{(2-m)}{(1-m)^{2}}|b|\left(\frac{T_{2}}{T_{1}}\right)^{2}\left\{\left(m^{2}-5 m+6\right)\left(3 T_{3}-1\right)+6 m^{2}-21 m+6\right\}\right. \\
& \left.-(m+1)|a|\left(\frac{Z_{2}}{Z_{1}}\right)^{2}\left(3 T_{3}+2\right)+4\left\{|a|\left(3 T_{3}-1\right)+\frac{3(2-m)}{(1-m)}|b|\right\}+3 \beta_{0}^{2}\left(3 T_{3}+2\right)\right], \\
& 32 \pi(\rho+3 p)=e^{-A}\left[\frac{(2-m)}{(1-m)^{2}}|b|\left(\frac{T_{2}}{T_{1}}\right)^{2}\left\{\left(m^{2}-5 m+6\right)\left(3 T_{3}+1\right)+6 m^{2}-21 m+6\right\}\right. \\
& \left.+(m+1)|a|\left(\frac{Z_{2}}{Z_{1}}\right)^{2}\left(3 T_{3}+4\right)+4\left\{|a|\left(3 T_{3}+1\right)+\frac{3(2-m)}{(1-m)}|b|\right\}-3 \beta_{0}^{2}\left(3 T_{3}+4\right)\right] .
\end{aligned}
$$

The reality conditions $\rho \geq 0, p \geq 0$ and $\rho-3 p \geq 0$ impose further restrictions on both of these models.

\section{B. Case II: Time varying displacement field $\left(\beta=\beta_{0} t^{\alpha}\right)$}

Using the aforesaid power law relation between time coordinate and displacement field, Eq. (19) may be written as

$$
\ddot{w}-\left[\frac{3(1-m) \beta_{0}^{2}}{4(2-m)^{2}} t^{2 \alpha}-\frac{k(1-m)}{(2-m)^{2}}\right] w=0,
$$

where

$$
w=e^{-\frac{(1-m)}{(2-m)}} B_{2} .
$$

Now, it is difficult to find a general solution of Eq. (39) and hence we consider a particular case of physical interest. It is believed that $\beta^{2}$ has similar behaviour as the cosmological constant which decreases during expansion of universe. Several authors [53]-[65] have considered the relation $\beta \sim \frac{1}{t}$ for study of cosmological models in different context. 
Considering $\alpha=-1, \beta=\frac{\beta_{0}}{t}$ and hence Eq. (39) reduces to

$$
t^{2} \ddot{w}+\left[\frac{k(1-m)}{(2-m)^{2}} t^{2}-\frac{3}{4} \frac{(1-m)}{(2-m)^{2}} \beta_{0}^{2}\right] w=0 .
$$

Eq. (41) yields the general solution

$$
w t^{r+1}=\left(t^{3} D\right)^{r}\left[\frac{c_{1} e^{h t}+c_{2} e^{-h t}}{t^{2 r-1}}\right]
$$

where

$$
\begin{aligned}
& D \equiv \frac{d}{d t} \\
& r=\frac{1}{2}\left[\left\{1+\frac{3(1-m)}{(2-m)^{2}} \beta_{0}^{2}\right\}^{\frac{1}{2}}-1\right] \\
& h^{2}=\frac{k(1-m)}{(2-m)^{2}}
\end{aligned}
$$

$$
\text { For } r=1, \beta_{0}^{2}=\frac{8(2-m)^{2}}{3(1-m)} \text {, Eq. (42) suggests }
$$

$$
w=\left(h-\frac{1}{t}\right) c_{3} e^{h t}-\left(h+\frac{1}{t}\right) c_{4} e^{-h t}
$$

where $c_{3}$ and $c_{4}$ are integrating constants.

Hence the metric coefficients have the explicit forms as

$$
e^{A}=\left\{c_{1} \sinh (z \sqrt{|a|})+c_{2} \cosh (z \sqrt{|a|})\right\}^{m} \times
$$

$$
\left[\left(h-\frac{1}{t}\right) c_{3} e^{h t}-\left(h+\frac{1}{t}\right) c_{4} e^{-h t}\right]^{(m-2)} \quad \text { when } a<0
$$

$$
\begin{gathered}
e^{B}=\left\{c_{1} \sinh (z \sqrt{|a|})+c_{2} \cosh (z \sqrt{|a|})\right\} \times \\
{\left[\left(h-\frac{1}{t}\right) c_{3} e^{h t}-\left(h+\frac{1}{t}\right) c_{4} e^{-h t}\right]^{-\frac{(2-m)}{(1-m)}} \quad \text { when } a<0}
\end{gathered}
$$

With the help of Eq. (44) and (45), the energy density and pressure can be obtained from Eqs. (7) and (8)

$$
\begin{gathered}
32 \pi \rho=e^{-A}\left[|a|\left\{\left(\frac{Z_{2}}{Z_{1}}\right)^{2}(1+m)-4\right\}+\frac{(3-m)(2-m)^{2}}{(1-m)^{2}}\left(\frac{c_{3} h^{2} t}{T_{5}}-\frac{1}{t}\right)^{2}-\frac{3 \beta_{0}^{2}}{t^{2}}\right] \quad \text { when } a<0 \\
32 \pi(p-\xi \theta)=e^{-A}\left[(m+1)|a|\left(\frac{Z_{2}}{Z_{1}}\right)^{2}-\frac{(1+2 m)(2-m)^{2}}{(1-m)^{2}}\left(\frac{c_{3} h^{2} t}{T_{5}}-\frac{1}{t}\right)^{2}-\right. \\
\left.\frac{4(2-m)}{(1-m)}\left\{\frac{1}{t^{2}}-\frac{4 c_{4} h^{3} t e^{-2 h t}}{T_{5}}+h^{2}\left(\frac{T_{4}}{T_{5}}\right)^{2}\right\}-\frac{3 \beta_{0}^{2}}{t^{2}}\right] \text { when } a<0
\end{gathered}
$$

where

$T_{4}=c_{3}+c_{4}(1+2 h t) e^{-2 h t}$

$T_{5}=c_{3}(h t-1)-c_{4}(1+h t) e^{-2 h t}$

From the above results in both cases it is evident that at any instant the domain wall density $\rho$ and pressure $p$ in the perpendicular direction decreases on both sides of the wall away from the symmetry plane and both vanish as $Z \longrightarrow \pm \infty$. The space times in both cases are reflection symmetry with respect to the wall. All these properties are very much expected for a domain wall.

The expression for kinematical parameter expansion $\theta$ is given by

$$
\theta=\frac{(m-2)(3-m)}{2(1-m)} e^{-\frac{A}{2}}\left[c_{3}\left(h^{2} t^{2}-h t+1\right)+c_{4} e^{-2 h t}\left(h^{2} t^{2}+h t+1\right)\right] .
$$

In this case we again consider the following two cases:

\section{Model I: solution for $\xi=\xi_{0}$}

When $n=0$, Eq. (28) reduces to $\xi=\xi_{0}=$ constant. Hence in this case Eq. (47), with the use of (48), leads to

$$
32 \pi p=e^{-A}\left[(m+1)|a|\left(\frac{Z_{2}}{Z_{1}}\right)^{2}+\frac{(1+2 m)(2-m)^{2}}{(1-m)^{2}}\left(\frac{c_{3} h t}{T_{5}}-\frac{1}{t}\right)-\right.
$$




$$
\left.\frac{4(2-m)}{(1-m)}\left\{\frac{1}{t^{2}}-\frac{4 c_{4} h^{3} t e^{-2 h t}}{T_{5}}+h^{2}\left(\frac{T_{4}}{T_{5}}\right)^{2}\right\}-\frac{3 \beta_{0}^{2}}{t^{2}}\right]+\frac{16 \pi e^{-\frac{A}{2}}(m-2)(3-m)}{(1-m)}\left(\frac{T_{6}}{T_{5}}\right),
$$

where $T_{6}=\left[c_{3}\left(h^{2} t^{2}-h t+1\right)+c_{4} e^{-2 h t}\left(h^{2} t^{2}+h t+1\right)\right] \xi_{0}$.

\section{Model II: solution for $\xi=\xi_{0} \rho$}

When $n=1$, Eq. (28) reduces to $\xi=\xi_{0} \rho$. Hence in this case Eq. (47), with the use of (48), leads to

$$
\begin{gathered}
32 \pi p=e^{-A}\left[(m+1)|a|\left(\frac{Z_{2}}{Z_{1}}\right)^{2}\left\{\frac{e^{-\frac{A}{2}}(m-2)(3-m)}{2(1-m)}\left(\frac{T_{6}}{T_{5}}\right)+1\right\}+\right. \\
\left(\frac{c_{3} h^{2} t}{T_{5}}-\frac{1}{t}\right)^{2} \frac{(m-2)}{(1-m)}\left\{\frac{e^{-\frac{A}{2}}(3-m)}{2}\left(\frac{T_{6}}{T_{5}}\right)-\frac{(1+2 m)(m-2)}{(1-m)}\right\} \\
\left.-\frac{3 \beta_{0}^{2}}{t^{2}}\left(T_{6}+1\right)+h^{2}\left(\frac{T_{4}}{T_{5}}\right)^{2}-\frac{4 c_{4} h^{3} t e^{-2 h t}}{T_{4}}\right]
\end{gathered}
$$

\section{Physical Consequences of the models}

From the above results in both cases it is evident that at any instant the domain wall density $\rho$ and pressure $p$ in the perpendicular direction decreases on both sides of the wall away from the symmetry plane and both vanish as $Z \longrightarrow \pm \infty$.
The space times in both cases are reflection symmetry with respect to the wall. All these properties are very much expected for a domain wall.

The weak and strong energy conditions, we have, in Model I

$$
\begin{gathered}
32 \pi(\rho-p)=e^{-A}\left[4\left(\frac{2-m}{1-m}\right)\left\{\frac{1}{t^{2}}-\frac{4 c_{4} h^{3} t e^{-2 h t}}{T_{5}}+h^{2}\left(\frac{T_{4}}{T_{5}}\right)^{2}\right\}-4|a|\right. \\
\left.-\frac{(2-m)^{2}(3 m-2)}{(1-m)^{2}}\left(\frac{c_{3} h^{2} t}{T_{5}}-\frac{1}{t}\right)^{2}\right]-\frac{16 \pi e^{-\frac{A}{2}(m-2)(3-m)}}{(1-m)}\left(\frac{T_{6}}{T_{5}}\right) \\
32 \pi(\rho+p)=e^{-A}\left[2(m+1)|a|\left(\frac{Z_{2}}{Z_{1}}\right)^{2}-4\left(\frac{2-m}{1-m}\right)\left\{\frac{1}{t^{2}}-\frac{4 c_{4} h^{3} t e^{-2 h t}}{T_{5}}+\right.\right. \\
\left.\left.h^{2}\left(\frac{T_{4}}{T_{5}}\right)^{2}\right\}+4|a|+\frac{(2-m)^{2}(m+4)}{(1-m)^{2}}\left(\frac{c_{3} h^{2} t}{T_{5}}-\frac{1}{t}\right)^{2}-\frac{6 \beta_{0}^{2}}{t^{2}}\right] \\
+\frac{16 \pi e^{-\frac{A}{2}}(m-2)(3-m)}{(1-m)}\left(\frac{T_{6}}{T_{5}}\right), \\
32 \pi(\rho-3 p)=e^{-A}\left[12\left(\frac{2-m}{1-m}\right)\left\{\frac{1}{t^{2}}-\frac{4 c_{4} h^{3} t e^{-2 h t}}{T_{5}}+h^{2}\left(\frac{T_{4}}{T_{5}}\right)^{2}\right\}-4|a|\right. \\
\left.-2(m+1)|a|\left(\frac{Z_{2}}{Z_{1}}\right)^{2}-\frac{7 m(2-m)^{2}}{(1-m)^{2}}\left(\frac{c_{3} h^{2} t}{T_{5}}-\frac{1}{t}\right)^{2}+\frac{6 \beta_{0}^{2}}{t^{2}}\right]
\end{gathered}
$$




$$
\begin{gathered}
-\frac{48 \pi e^{-\frac{A}{2}}(m-2)(3-m)}{(1-m)}\left(\frac{T_{6}}{T_{5}}\right) \\
32 \pi(\rho+3 p)=e^{-A}\left[4(m+1)|a|\left(\frac{Z_{2}}{Z_{1}}\right)^{2}-12\left(\frac{2-m}{1-m}\right)\left\{\frac{1}{t^{2}}-\frac{4 c_{4} h^{3} t e^{-2 h t}}{T_{5}}+\right.\right. \\
\left.\left.h^{2}\left(\frac{T_{4}}{T_{5}}\right)^{2}+4|a|\right\}+\frac{(2-m)^{2}(5 m+6)}{(1-m)^{2}}\left(\frac{c_{3} h^{2} t}{T_{5}}-\frac{1}{t}\right)^{2}-\frac{12 \beta_{0}^{2}}{t^{2}}\right] \\
+\frac{48 \pi e^{-\frac{A}{2}}(m-2)(3-m)}{(1-m)}\left(\frac{T_{6}}{T_{5}}\right) .
\end{gathered}
$$

In Model II, we have

$$
\begin{aligned}
& 32 \pi(\rho-p)=e^{-A}\left[( \frac { 2 - m } { 1 - m } ) ( \frac { c _ { 3 } h ^ { 2 } t } { T _ { 5 } } - \frac { 1 } { t } ) ^ { 2 } \left\{\frac{3-m}{(1-m)^{2}}+\frac{e^{-\frac{A}{2}}(3-m)}{2}\left(\frac{T_{6}}{T_{5}}\right)+\right.\right. \\
& \left.\frac{(1+2 m)(m-2)}{(1-m)}\right\}+\frac{3 \beta_{0}^{2}}{t^{2}} T_{6}-(m+1)|a|\left(\frac{Z_{2}}{Z_{1}}\right)^{2} \frac{e^{-\frac{A}{2}}(m-2)(3-m)}{2(1-m)}\left(\frac{T_{6}}{T_{5}}\right) \\
& \left.-h^{2}\left(\frac{T_{4}}{T_{5}}\right)^{2}+4\left\{\frac{c_{4} h^{3} t e^{-2 h t}}{T_{5}}-|a|\right\}\right] \\
& 32 \pi(\rho+p)=e^{-A}\left[(m+1)|a|\left(\frac{Z_{2}}{Z_{1}}\right)^{2}\left\{\frac{e^{-\frac{A}{2}}(3-m)(m-2)}{2(1-m)}\left(\frac{T_{6}}{T_{5}}\right)+2\right\}\right. \\
& +\left(\frac{2-m}{1-m}\right)\left(\frac{c_{3} h^{2} t}{T_{5}}-\frac{1}{t}\right)^{2}\left\{\frac{3-m}{1-m}-\frac{e^{-\frac{A}{2}}(3-m)}{2}\left(\frac{T_{6}}{T_{5}}\right)+\frac{(1+2 m)(m-2)}{(1-m)}\right\} \\
& \left.-\frac{3 \beta_{0}^{2}}{t^{2}}\left(T_{5}+2\right)+h^{2}\left(\frac{T_{4}}{T_{5}}\right)^{2}-4\left\{\frac{c_{4} h^{3} t e^{-2 h t}}{T_{5}}+|a|\right\}\right] \\
& 32 \pi(\rho-3 p)=e^{-A}\left[( \frac { 2 - m } { 1 - m } ) ( \frac { c _ { 3 } h ^ { 2 } t } { T _ { 5 } } - \frac { 1 } { t } ) ^ { 2 } \left\{\frac{3-m}{1-m}+\frac{e^{-\frac{A}{2}}(3-m)}{2}\left(\frac{T_{6}}{T_{5}}\right)\right.\right. \\
& \left.-\frac{3(1+2 m)(m-2)}{(1-m)}\right\}+\frac{3 \beta_{0}^{2}}{t^{2}}\left(3 T_{6}+2\right)-3 h^{2}\left(\frac{T_{4}}{T_{5}}\right)^{2} \\
& \left.+4\left\{\frac{c_{4} h^{3} t e^{-2 h t}}{T_{5}}-|a|\right\}\right] \\
& 32 \pi(\rho+3 p)=e^{-A}\left[(m+1)|a|\left(\frac{Z_{2}}{Z_{1}}\right)^{2}\left\{\frac{e^{-\frac{A}{2}}(3-m)(m-2)}{2(1-m)}\left(\frac{3 T_{6}}{T_{5}}\right)+4\right\}\right. \\
& +\left(\frac{2-m}{1-m}\right)\left(\frac{c_{3} h^{2} t}{T_{5}}-\frac{1}{t}\right)^{2}\left\{\frac{3-m}{1-m}-\frac{e^{-\frac{A}{2}}(3-m)}{2}\left(\frac{3 T_{6}}{T_{5}}\right)+\right. \\
& \left.\left.\frac{3(1+2 m)(m-2)}{(1-m)}\right\}-\frac{3 \beta_{0}^{2}}{t^{2}}\left(3 T_{6}+4\right)+3 h^{2}\left(\frac{T_{4}}{T_{5}}\right)^{2}-4\left\{\frac{c_{4} h^{3} t e^{-2 h t}}{T_{5}}+|a|\right\}\right] .
\end{aligned}
$$

The reality conditions $\rho \geq 0, p \geq 0$ and $\rho-3 p \geq 0$ impose further restrictions on both of these models.

\section{STUDY OF GEODESICS}

The trajectory of the test particle $x^{i}\{t(\lambda), x(\lambda), y(\lambda), z(\lambda)\}$ in the gravitational field of domain wall can be determined by integrating the geodesic equations

$$
\frac{d^{2} x^{\mu}}{d \lambda^{2}}+\Gamma_{\alpha \beta}^{\mu} \frac{d x^{\alpha}}{d \lambda} \frac{d x^{\beta}}{d \lambda}=0
$$


for the metric (5). It has been already mentioned in [23], the acceleration of the test particle in the direction perpendicular to the domain wall ( i.e. in the z-direction) may be expressed as

$$
\ddot{z}=\frac{e^{B-A}}{2} \frac{\partial B}{\partial z}\left(\dot{x}^{2}+\dot{y}^{2}\right)-\frac{1}{2} \frac{\partial A}{\partial z}\left(\dot{t}^{2}+\dot{z}^{2}\right)-\frac{\partial A}{\partial z} \dot{t} \dot{z}
$$

By simple but lengthy calculation, one can get expression for acceleration which may be positive, negative (or zero) depending on suitable choice of the constants. This implies that the gravitational field of domain wall may be repulsive or attractive in nature, or without a gravitational effect.

\section{CONCLUSIONS}

The present study deals with plane symmetric domain wall within the framework of Lyra geometry, in presence of bulk viscous fluid. The essential difference between the cosmological theories based on Lyra geometry and Riemannian geometry lies in the fact that the constant vector displacement field $\beta$ arises naturally from the concept of gauge in Lyra geometry, whereas the cosmological constant $\Lambda$ was introduced in ad hoc fashion in the usual treatment. Currently the study of domain walls and cosmological constant have gained renewed interest due to their application in structure formation in the Universe. Recently Rahaman et al [23] have presented a cosmological model for domain wall in Lyra geometry under a specific condition by taking displacement fields $\beta$ as constant. The cosmological models based on varying displacement vector field $\beta$ have widely been considered in the literature in different context [38]-[42]. Motivated by these studies it is worthwhile to consider domain walls with a time varying $\beta$ in Lyra geometry. In this paper both cases viz., constant and time varying displacement field $\beta$, are discussed in the context of domain walls with the framework of Lyra geometry. It has been pointed out that the result of Rahman et al [23] is a special case of our results.

The study on domain wall in this article successfully describes the various features of the Universe. The effect of bulk viscosity is to produce a change in perfect fluid and hence exhibit essential influence on the character of the solution. We observe here that Murphy's conclusion [50] about the absence of a big bang type singularity in the infinite past in models with bulk viscous fluid is, in general, not true. The results obtained in [20] also show that, it is, in general, not valid, since for some cases big bang singularity occurs in finite past.

\section{Acknowledgements}

The authors (AP and AKY) would like to thank the HarishChandra Research Institute, Allahabad, India for hospitality where part of this work is done.
[1] A. Vilenkin and E. P. S. Shellard, Cosmic string and other topological defects ( Cambridge: Cambridge University Press, 1994).

[2] T. W. B. Kibble, J. Phys. A 9, 1387 (1976); Phys. Rep. 67, 183 (1980).

[3] Ya. B. Zel'dovich, I. Yu. Kobzarev, and L. B. Okun, Zh. Eksp. Teor. Fiz. 67, 3 (1974) [ Sov. Phys. JETP 40, 1 (1975)].

[4] W. B. Kibble, G. Lazarides, and Q. Shafi, Phys. Rev. D 26, 435 (1982);

A. Vilenkin and A. E. Everett, Phys. Rev. Lett. 48, 1867 (1982); A. E. Everett and A. Vilenkin, Nucl. Phys. B 207, 43 (1982).

[5] C. T. Hill, D. N. Schramm, and J. N. Fry, Nucl. Phys. B 111, 253 (1988).

[6] L. M. Widrow, Phys. Rev. D 39, 3571 (1989).

[7] A. Vilenkin, Phys. Lett. B 133, 177 (1983).

[8] J. Ipser and P. Sikivie, Phys. Rev. D 30, 712 (1984).

[9] W. Israel, Nuovo Cim. B 44 , 1 (1966).

[10] D. Garfinkle and R. Gregory, Phys. Rev. D 41, 1889 (1990).

[11] C. T. Hill, D. N. Schramm, and J. N. Fry, Comment Nucl. Part. Phys. 19, 25 (1989).

[12] G. Goetz, J. Math. Phys. 31, 2683 (1990).

[13] M. Mukherjee, Class. Quant. Grav. 10, 131 (1993).

[14] F. Bonjour, C. Charmousis, and R. Gregory, [qr-qc/9903059] talk given at Les Houches Winter School (1999).

[15] F. Bonjour, C. Charmousis, and R. Gregory, Class. Quant. Grav. 16, 2427 (1999)
[16] B. Jensen and H. H. Soleng, Class. Quant. Grav. 14, 1821 (1997).

[17] E. W. Kolb and M. S. Turner, The Early Universe (Addison Wesley, U S A, 1990).

[18] S. Myung and B. M. Cho, Mod. Phys. Letter A 1, 37 (1987).

[19] N. Turok, Phys. Rev. Lett. 60, 549 (1988).

[20] J. D. Barrow, Nucl. Phys. B 310, 243 (1988).

[21] C. Wolf, S.-Afr. Tydskr. 14, 68 (1991).

[22] Ø. Grøn, Astrophys. Space Sci. 173, 191 (1990).

[23] F. Rahaman, S. Chakraborty, and M. Kalam, Int. J. Mod. Phys. D 10, 735 (2001).

[24] G. Lyra, Math. Z. 54, 52 (1951).

[25] H. Weyl, Sber. Preuss. Akad. Wiss. Berlin, 465 (1918).

[26] D. K. Sen, Z. Phys. 149, 311 (1957).

[27] D. K. Sen and K. A. Dunn, J. Math. Phys. 12, 578 (1971).

[28] W. D. Halford, Austr. J. Phys. 23, 863 (1970).

[29] W. D. Halford, J. Math. Phys. 13, 1399 (1972).

[30] D. K. Sen and J. R. Vanstone, J. Math. Phys. 13, 990 (1972).

[31] K. S. Bhamra, Austr. J. Phys. 27, 541 (1974).

[32] T. M. Karade and S. M. Borikar, Gen. Rel. Gravit. 9, 431 (1978).

[33] S. B. Kalyanshetti and B. B. Waghmode, Gen. Rel. Gravit. 14, 823 (1982)

[34] D. R. K. Reddy and P. Innaiah, Astrophys. Space Sci. 123, 49 (1986).

[35] A. Beesham, Astrophys. Space Sci. 127, 189 (1986); Austr. J. 
Phys. 41, 833 (1988).

[36] D. R. K. Reddy and R. Venkateswarlu, Astrophys. Space Sci. 136, 191 (1987).

[37] H. H. Soleng, Gen. Rel. Gravit. 19, 1213 (1987).

[38] T. Singh and G. P. Singh, J. Math. Phys. 32, 2456 (1991a); Il. Nuovo Cimento B 106, 617 (1991b); Int. J. Theor. Phys. 31, 1433 (1992); Fortschr. Phys. 41, 737 (1993).

[39] G. P. Singh and K. Desikan, Pramana 49, 205 (1997).

[40] A. Pradhan and A. K. Vishwakarma, J. Geom. Phys. 49, 332 (2004).

[41] A. Pradhan, V. K. Yadav, and I. Chakrabarty, Int. J. Mod. Phys. D 10, 339 (2000).

[42] A. Pradhan, L. Yadav, and A. K. Yadav, Astrophys. Space Sci. 299, 31 (2005)

[43] F. Hoyle, Monthly Notices Roy. Astron. Soc. 108, 252 (1948).

[44] F. Hoyle and J. V. Narlikar, Proc. Roy. Soc. London Ser. A 273, 1 (1963).

[45] F. Hoyle and J. V. Narlikar, Proc. Roy. Soc. London Ser. A 282, 1 (1964).

[46] A. H. Taub, Ann. Math. 53, 472 (1951).

[47] D. Pavon, J. Bafaluy, and D. Jou, Class. Quant. Grav. 8, 357 (1991); in Proc. Hanno Rund Conf. on Relativity and Thermodynamics (Ed. S. D. Maharaj), University of Natal, Durban, 1996, p. 21

[48] R. Maartens, Class. Quant. Grav. 12, 1455 (1995).

[49] W. Zimdahl, Phys. Rev. D 53, 5483 (1996).
[50] G. L. Murphy, Phys. Rev. D 8, 4231 (1973).

[51] S. Weinberg, Graviatation and Cosmology, Wiley, New York, (1972), p. 57

[52] N. O. Santos, R. S. Dias, and A. Banerjee, J. Math. Phys., 26, 878 (1985).

[53] M. S. Berman and M. M. Som, Int. J. Theor. Phys. 29, 1311 (1990).

[54] M. S. Berman, M. M. Som, and F. M. Gomide, Gen. Rel. Grav. 21, 287 (1989).

[55] M. S. Berman, Int. J. Theor. Phys. 29, 567 (1990).

[56] M. S. Berman, Int. J. Theor. Phys. 29, 1419 (1990).

[57] O. Bertolami, Nuovo Cimento 39, 36 (1986).

[58] O. Bertolami, Fortschr. Phys. 34, 829 (1986).

[59] A. Beesham, Phys. Rev. D 48, 3539 (1993).

[60] A. Beesham, Int. J. Theor. Phys. 25, 1295 (1986).

[61] T. Singh, A. Beesham, and W. S. Mbokazi, Gen. Rel. Grav. 30, 537 (1998).

[62] A. Pradhan and A. K. Vishwakarma, SUJST, XII, Sec.B, 42 (2000).

[63] A. Pradhan and A. Kumar, Int. J. Mod. Phys. D 10, 291 (2001).

[64] I. Chakrabarty and A. Pradhan, Gravitation \& Cosmology 7, 55 (2001).

[65] A. Pradhan, N. N. Saste, and V. K. Yadav, Int. J. Mod. Phys. D 11, 857 (2002). 